\title{
Qualitative Risk Assessments in Project Management in Construction Industry
}

\author{
Vladimir Burkov ${ }^{1}$, Irina Burkova $^{l^{*}}$, Reza Barkhi $^{2}$ and Mikhail Berlinov ${ }^{3}$ \\ ${ }^{1}$ V.A. Trapeznikov Institute of Control Sciences of Russian Academy of Sciences, st.Profsouznaya, \\ 65, Moscow, 117997, Russia \\ ${ }^{2}$ Virgina Tech University, Pamplin 3090, 880 Campus Drive, Blacksburg, VA 24061, USA \\ ${ }^{3}$ Moscow State University of Civil Engineering, Yaroslavskoe shosse, 26, Moscow, 129337, Russia
}

\begin{abstract}
Modern economic conditions require construction companies to promptly prevent, identify and manage risks in various areas of their construction activities. As this research shows, many of the best practices of corporate risk management are adopted and applied in large Russian construction companies and in various industries. At the same time, the results of the research demonstrate the possibilities for further development. Key areas of development include: the process of integrating risk management into the development strategy of a construction company, the collection and accumulation of statistical data, interaction between units, the participation and active participation of key stakeholders, and the quality of information on key risks. Risk management is one of the most important functional areas in the methodology of project management in the construction sector. In practice, as a rule, in addition to quantitative risk assessment, qualitative risk assessments in construction is also always utilized. However, the theory of risk management based on qualitative assessments is virtually absent in the Russian practice. This article provides a definition of qualitative risk assessments and complex risks in the construction field. We desceibe the tasks involved in risk management in construction and propose algorithms for solving corresponding problems in the construction field.
\end{abstract}

\section{Introduction}

From an investor's point of view, any project is a risk. Construction is a special category of facilities, which carries an even higher level of risks. Much depends on the geographical features of the construction region, the level of social development, the mentality of the population and many other factors, that are identified as project risks.

Countries that have little experience in construction and high-rise construction can fuel growth and development of the construction industry, and can enhance the standard of living for the citizens. At the same time, each country has its own peculiarities, which determine the risks, potential value created by proper construction, and the probability of losses for the investor should the construction project risks and not properly managed.

\footnotetext{
* Corresponding author: ibur27@gmail.com
} 
The cost of construction varies in different countries and depends largely on the project risks [1].

For instance, one of the important factors that determines the cost of construction of buildings include the climatic conditions. Ground movements, the influence of water, and wind load complicate the project and cause a significant rise in price not only at the design stage of the object, but also during construction. The geography of the country largely determines the level of risks and potential natural and climatic threats. Nevertheless, risks associated with the socio-political and economic level of development should also be taken into account.

Therefore, the problem of risks can be considered as a price factor that affects the investment cost of construction to a certain extent. The most likely and common types of risks are considered in order to adequately evaluate the risks as a practical tool for assessing the level of danger for a high-rise facility.

Several researchers such as John Creswel, Vladimir N. Burkov, Dmitry A. Novikov, Alexander V. Shchepkin, Victoria Borkovskaya have focused on the problem of risk evaluation within construction field. Their body of research provide a risk evaluation methodology which includes the analysis of the construction industry in terms of various options for implementing investment projects. The issues of classification, analysis and assessment of risks of construction require a focus on construction specific issues to make the risk based approach to construction industry relevant to the practice of construction.

The goal of this study is to develop a risk analysis model that allows determining the investment value of risk taking into account the probability of a risk event and possible consequences $[2,3]$.

\section{Methodology}

To determine qualitative risk assessments, we define two scales: 1) the scale of probabilities, and 2) the scale of damages. We confine ourselves to the consideration of two-price scales. On the scale of probabilities we define expertly the boundary point v. If the project has a low probability risk, it means that the probability $\mathrm{p}$ of the risk event is less than or equal to v. If the project has a high risk, then $\mathrm{p}>\mathrm{v}$. Similarly, we introduce the boundary point $\mathrm{u}$ for the damage (the fraction of the cost of the project). If the damage is low, then it is less or equal to u, otherwise the damage is high. Since we do not know anything about probability and damage distribution functions at low and high risk intervals, we assume that they are uniform. Define the base point

for probability of risk and

$$
v_{1}=\frac{v}{2}, v_{2}=\frac{1+v}{2}
$$

$$
u_{1}=\frac{u}{2}, v_{2}=\frac{u_{m}+u}{2}
$$

for damage $\left(\boldsymbol{u}_{\boldsymbol{m}}\right.$ is the coefficient determining how many times the maximum damage is less or more than the cost of the project, respectively, if you designate the cost of the project $\mathrm{c}$, then the maximum damage amount will be $\boldsymbol{u}_{\boldsymbol{m}} \boldsymbol{c}$ ). For definiteness, we take $\boldsymbol{u}_{\boldsymbol{m}}=$ 1 ; The maximum damage does not exceed the cost of the project, although the opposite happens.

Now we can determine the boundary level of the expected damage (i.e. the degree of influence): $\boldsymbol{w}=\boldsymbol{v} \boldsymbol{u}$ and its base levels $\boldsymbol{w}_{\mathbf{1}}=\boldsymbol{v}_{\mathbf{1}} \boldsymbol{u}_{\mathbf{1}}$ and $\boldsymbol{w}_{\mathbf{2}}=\boldsymbol{v}_{\mathbf{2}} \boldsymbol{u}_{\mathbf{2}}$. There are two options for qualitative assessments - a low value of the indicator $(\mathrm{H})$ and a high one (B). For any situation, the expected damage can be described by a pair, where the probability rating is in the first place, and the damage on the second. There are four combinations of 
qualitative assessments: $(\mathrm{HH}),(\mathrm{HB}),(\mathrm{HH})$, and $(\mathrm{BB})$. Obviously, the variant $(\mathrm{LV})$ has a low degree of influence, (BB) - high. To determine the degree of influence of options $(\mathrm{HB})$ and $(\mathrm{BH})$, proceed as follows. If for option (HB) $\boldsymbol{v}_{\mathbf{1}} \boldsymbol{u}_{\mathbf{2}} \leq \boldsymbol{v} \boldsymbol{u}$, then the option has a low degree of influence, otherwise - high. For the variant $(\mathrm{BH})$ we shall consider the product $\boldsymbol{v}_{\mathbf{2}} \boldsymbol{u}_{\mathbf{1}}$ in a similar way [4].

We proceed to assess complex risks. Let the project consist of $\mathrm{n}$ papers. We will consider the risks of works as independent random variables. In this case, the damage is summarized. For each work i, let us denote the three qualitative estimates - $\left(\boldsymbol{x}_{\boldsymbol{i}}, \boldsymbol{y}_{\boldsymbol{i}}, \boldsymbol{z}_{\boldsymbol{i}}\right)$, where $\boldsymbol{x}$ is the probability estimate, $\boldsymbol{y}$ is the damage estimate, and $\boldsymbol{z}$ is the degree of influence of the $\boldsymbol{i}$-th work. Since the expected damages of independent random variables are summed up, a qualitative assessment of the degree of project impact is defined as follows. Let $\boldsymbol{Q}_{\mathbf{1}}$ denote the set of works with a low estimate both in probability and in damage, $\boldsymbol{Q}_{\mathbf{2}}$ is the set of works with a low probability and low damage estimate, $\boldsymbol{Q}_{3}$ is the set of works with a high probability and low damage estimate, $\boldsymbol{Q}_{\mathbf{4}}$ is the set of works with high both in probability and in damage:

$$
a_{i}=\frac{c}{c_{i}}, \quad i=\overline{1,4}
$$

where $\boldsymbol{c}_{\boldsymbol{i}}$ is the cost of the works of $\boldsymbol{Q}_{\boldsymbol{i}}, \boldsymbol{c}$ is the sum of the cost of the work. Calculate the expected damage per unit of cost

$$
\begin{gathered}
W=a_{1} u_{1} v_{1}+a_{2} u_{2} v_{1}+a_{3} u_{1} v_{2}+a_{4} u_{2} v_{2}, \\
U=a_{1} u_{1}+a_{2} u_{2}+a_{3} u_{1}+a_{4} u_{2},
\end{gathered}
$$

and probability

$$
V=\frac{W}{U}
$$

On the appropriate scales we determine the qualitative risk assessments of the project as a whole.

\subsection{Project Risk Management. Risk reduction strategy}

Reduction of risk is understood as a reduction in the degree of influence of project risks by carrying out activities aimed at reducing the likelihood of a risk event and/or reducing the damage when it occurs and, accordingly, reducing the degree of influence. Note that for works of type HB or HV there is only one option to reduce the degree of influence. For works of the type HB, this reduction in damage to a low level, and for works of the HV type this reduces the probability to a low level. For works of type BB, there are three options for reducing the degree of influence. The first is to translate the work from BB to type $\mathrm{HB}$, the second to translate into $\mathrm{BH}$ type and the third into HH type [5-7].

For a formal statement of the problem, we introduce the notation. For works of type $\mathrm{HB}$ or $\mathrm{BH}$, we denote $x_{i}=1$, if measures are taken to reduce the risk with costs $s_{i}, x_{i}=0$ otherwise. For works of type BB, we denote $x_{i j}=1$ if for option $i$ is selected option $j$, and $j=1$ means the transfer of works to type HB;

$j=2$ means the transfer of works to the $\mathrm{BH}$ type;

$j=3$ means the translation of the work into the HH type;

Accordingly, we denote $\boldsymbol{s}_{i j}$ - costs in variant $j$.

Determine the degree of impact of project risks for given $x$.

We denote by $w_{11}=v_{1} u_{1}, w_{12}=v_{1} u_{2}, w_{21}=v_{2} u_{1}, w_{22}=v_{2} u_{2}, n_{1}$ - number of works set $Q_{1}, w_{0}$ - existing level of influence.

The task of reducing risk. We define $x$ such that 


$$
s(x)=\sum_{i \in Q_{2} \cup Q_{3}} s_{i} x_{i}+\sum_{i \in Q_{4}} \sum_{j} s_{i j} x_{i j} \rightarrow \min
$$

With restriction (8)

Where

$$
\alpha_{2} \sum_{i \in Q_{2}} \Delta_{12} x_{i}+\alpha_{3} \sum_{i \in Q_{3}} \Delta_{21} x_{i}+\alpha_{4} \sum_{i \in Q_{4}} \sum_{j} \Delta_{j} x_{i j} \geq w_{0}-w=\Delta
$$

$$
\Delta_{12}=w_{12}-w_{11}, \Delta_{21}=w_{21}-w_{11}, \Delta_{1}=w_{22}-w_{12}, \Delta_{2}=w_{22}-w_{21}, \Delta_{3}=w_{22}-w_{11} .
$$

The problem is some modification of the knapsack problem and is effectively solved by the method of dichotomous programming $[1,5]$.

\subsection{Risk management program. The strategy of evasion}

The strategy of evasion is applied mainly in the management of program risks in construction, or more precisely, - in the formation of the program's subject area (the set of projects included in the program). The essence of the evasion strategy is to limit the number of high-risk construction projects included in the program, by restricting their financing.

Consider the formulation of the problem. There are $n$ construction projects - applicants for inclusion in the program. Each project is characterized by the effect of $a_{i}$ on its implementation, the costs of implementing $c_{i}$ and qualitative risk assessments $(\mathrm{HH}, \mathrm{HB}, \mathrm{BH}$ or $\mathrm{BB})[8,9]$.

Denote $x_{i}=1$ if project $i$ is included in the program, $x_{i}=0$ otherwise.

Task 1. Identify $x=\left\{x_{i}, i=\overline{1, n}\right\}$ such that

$$
A(x)=\sum_{i} a_{i} x_{i} \rightarrow \max
$$

under constraints

$$
\begin{gathered}
\sum_{i} c_{i} x_{i} \leq R \\
\sum_{i} \alpha_{i} w_{i} x_{i} \leq R
\end{gathered}
$$

where $\mathrm{R}$-the amount of resources allocated to the program.

This is a two-dimensional knapsack problem, which is NP -hard and does not have effective methods of solution. We will consider another problem, close in meaning to problem 1, but effectively solved by the method of network programming. We denote QB the set of projects having a high level of influence, $\mathrm{R}_{\mathrm{B}}$ - the funds allocated for financing high-risk projects of the set Qв.

Task 2. Identify $x=\left\{x_{i}, i=\overline{1, n}\right\}$ such that

Under constraints

$$
A(x)=\sum_{i} a_{i} x_{i} \rightarrow \max
$$

$$
\begin{aligned}
& \sum_{i} c_{i} x_{i} \leq R, \\
& \sum_{i \in Q_{B}} c_{i} x_{i} \leq R_{B} .
\end{aligned}
$$

\section{Results and Discussion}

This document presents a model of the project management system in the area of construction, which allows for better identification and management of risks throughout the 
qualitative risk assessments. It shows the most popular methods with results for assessing the likelihood of risk and tries to indicate the advantages of a reliable approach to traditional methods. The modern development of project management, as well as the accumulated knowledge and experience in this field have allowed knowledge of project management to be integrated into a single model of the system. Within this model, standard and reliable approaches to the tasks of analyzing project data are applied and expanded.

Mixed risk management strategy. Let the mixed strategy of risk management in construction be chosen, when both risk reduction and avoidance are applied. Consider the approach to solving the problem, which is that solving problem 2 for different values of $\mathbf{R}_{b}$, we solve the problem of risk reduction every time when condition (12) is not fulfilled. All variants of different $\mathrm{R}_{6}$ are compared and the best effect is selected, minus the cost of risk reduction [6-10].

\section{Conclusions}

The methodology considered in the article allows enterprises to solve the tasks of risk management on the basis of risk reduction strategies and risk evasion using only qualitative assessments of risk characteristics (probability, damage, degree of influence). It is not difficult to generalize it to the case of a three-point or more scale. The formulation of problems and methods for their solution are preserved, however, of course, the computational complexity increases. So instead of four types of work. For a three-point scale there are already nine. These generalizations will be considered in subsequent publications [11-15].

\section{References}

1. Borkovskaya V.G. Economic cooperation in housing maintenance organizations. Dissertation. Moscow 2002.

2. Burkova I.V. The method of network programming in problems of nonlinear optimization. I.V. Burkova // Automation and telemechanics. - 2009.-No. 10.-P.15-21.

3. Burkov V.N., Novikov D.A., Shchepkin A.V. (2015) Simulation Models for Control Mechanisms in Ecological-Economic Systems. In: Control Mechanisms for Ecological-Economic Systems. Studies in Systems, Decision and Control, vol 10. Springer, Cham.

4. Borkovskaya V.G. Complex models of active control systems at the modern developing enterprises. Advanced Materials Research (Volumes 945-949). Chapter 22: Manufacturing Management and Engineering Management. June 2014. Pages 30123015. DOI: 10.4028/www.scientific.net/AMR.945-949.3012.

5. Borkovskaya V.G. The concept of innovation for sustainable development in the construction business and education. Applied Mechanics and Materials. (Volumes 475476). Chapter 15: Engineering Management. December 2013. Pages 1703-1706. DOI: 10.4028/www.scientific.net/AMM.475-476.1703.

6. Boris Titarenko, Amir Hasnaoui, Roman Titarenko. Risk management system model for construction projects. IOP Conference Series: Materials Science and Engineering, Volume 365, Safety in Construction.

7. BorkovskayaV.G. Bardenwerper W. Roe R. Interactive Teaching of Risk Management in the Russian Construction Industry. IOP Conf. Series: Materials Science and Engineering 365 (2018) 062030 doi:10.1088/1757-899X/365/6/062030.

8. Victoria G Borkovskaya, Evgeniy Degaev, Irina Burkova. Environmental economic model of risk management and costs in the framework of the quality management 
system. MATEC Web of Conference 2018, International Scientific Conference Environmental Science for Construction Industry - ESCI 2018.Volume 193 (2018). Ho Chi Minh City, Vietnam, March 2-5, 2018.

9. Leitch, M. "The Risk Management Gap", Board Leadership, vol. 2017, no. 154, pp. 13. doi:10.1002/bl.30088.

10. BorkovskayaV.G, Passmore D. Application of Failure Mode and Effects Analysis in Ecology in Russia. MATEC Web of Conference 2018, International Scientific Conference Environmental Science for Construction Industry - ESCI 2018.Volume 193 (2018). Ho Chi Minh City, Vietnam, March 2-5, 2018.

11. Victoria G Borkovskaya, Walter Bardenwerper, Richard Roe. Sustainability Risk Management: The Case for Using Interactive Methodologies for Teaching, Training and Practice in Environmental Engineering and Other Fields. Advances in Economics, Business and Management Research (France-Netherlands). Atlantis Press. In press.

12. BorkovskayaV.G, Passmore D. Behavioral engineering model to identify risks of losses in the construction industry. Advances in Economics, Business and Management Research (France-Netherlands). Atlantis Press. In press.

13. PMI 2017 A guide to the Project Management Body of Knowledge (PMBOK Guide) 6 (USA: PMI).

14. Degaev E, Suvorova A and Suhova A. Influence of total head of foam on optimum intensity and minimum particular expense solution size of foamer // IOP Conf. Ser.: Mater. Sci. Eng. 365 (2018) 032019. DOI: https://doi.org/10.1088/1757899X/365/3/032019.

15. Degaev E, Razvalyaeva V and Sabenina S. Formation of water film from aqueous film forming foam drops on the surface of oil products // IOP Conf. Ser.: Mater. Sci. Eng. 365 (2018) 062037. DOI: https://doi.org/10.1088/1757-899X/365/6/062037. 